\title{
SELF-HEATING OF CORONA BY ELECTROSTATIC FIELDS DRIVEN BY SHEARED FLOWS
}

\author{
H. SALEEM ${ }^{1}$, S. Ali ${ }^{1}$, AND S. PoedTs ${ }^{2}$ \\ ${ }^{1}$ National Centre for Physics, Shahdra Valley Road, Quaid-i-Azam University Campus, Islamabad 44000, Pakistan \\ ${ }^{2}$ K. U. Leuven, Centre for Plasma Astrophysics, and Leuven Mathematical Modeling and Computational Science Center (LMCC), \\ Celestijnenlaan 200B, 3001 Leuven, Belgium \\ Received 2011 September 5; accepted 2011 October 25; published 2012 March 13
}

\begin{abstract}
A mechanism for self-heating of the solar corona is discussed. It is shown that the free energy available in the form of sheared flows gives rise to unstable electrostatic perturbations which accelerate and heat particles. The electrostatic perturbations can occur through two processes, viz., by a purely growing sheared flow-driven instability and/or by a sheared flow-driven drift wave. These processes can occur throughout the corona and, hence, this self-heating mechanism could be very important for coronal heating. These instabilities can give rise to local perturbed electrostatic potentials $\varphi_{1}$ of up to 100 volts within $3 \times 10^{-2}$ to a few seconds time, if the (dimensionless) initial perturbation is assumed to be about $1 \%$, that is, $e \varphi_{1} / T_{e} \simeq 10^{-2}$. The wavelengths in the direction perpendicular to the external magnetic field $\mathbf{B}_{0}$ vary from about $10 \mathrm{~m}$ to $1 \mathrm{~m}$ in our model. The purely growing instability creates electrostatic fields by sheared flows even if there is no density gradient, whereas a density gradient is crucial for the occurrence of the drift wave instability. The purely growing instability develops a small real frequency as well in the two-ion coronal plasma. In the solar corona, very low frequency (of the order of $1 \mathrm{~Hz}$ ) drift dissipative waves can also occur due to electron-ion collisions.
\end{abstract}

Key words: instabilities - Sun: corona - waves

Online-only material: color figures

\section{INTRODUCTION}

Many theoretical models have been presented to explain the cause of solar coronal heating (see, e.g., the reviews by Aschwanden 2001; Mandrini et al. 2000). It is a well-known, though counterintuitive, fact that the corona of the Sun is about 200 times hotter than the lower lying chromosphere, while it contains a rarefied collisionless plasma with electron temperature $T_{e} \simeq 10^{6} \mathrm{~K}$. There are many unanswered questions in the problem of coronal heating. The temperature rises by two orders of magnitude from the upper chromosphere to lower corona through a narrow transition layer of only about $500 \mathrm{~km}$ (Priest 1982; Narain \& Ulmschneider 1990; Klimtchuk 2006). The proposed wave heating mechanisms assume that the waves originate in the lower regions, viz., the photosphere or even the convection layer of the Sun, and propagate through the transition region to deposit their energy in the corona. Observational data of Skylab, Yohkoh, the Solar and Heliospheric Observatory $(\mathrm{SOHO})$, and the Transition Region and Coronal Explorer (TRACE) have indicated that the entire corona is filled with open and closed magnetic field and only a subset of it is loaded with extremely hot plasma at a given time (Litwin \& Rosner 1993; Hara et al. 1992; Moses et al. 1997; Schrijver et al. 1999).

The coronal loops, omnipresent in active regions, have been considered to be responsible for localized heating. The bright coronal loops have higher densities compared to the ambient faint coronal plasma, which indicates that the heated plasma originates from the dense chromosphere. The coronal heating by acoustic waves originating from global oscillations has been ruled out (Aschwanden 2001). Alfvén waves have been considered to be the best candidate for carrying adequate energy fluxes from the chromosphere to the corona (Hollweg \& Sterling 1994). A great deal of work on Alfvén wave heating of the corona has appeared in the literature (Ionson 1983; Mok 1987; Steinolfson \& Davila 1993; Ofman et al. 1995; Poedts et al.
1989, 1990, 1994; Poedts \& Goedbloed 1997; Halberstadt \& Goedbloed 1995; Ruderman et al. 1997).

In fact, any unstable wave in the coronal plasma can cause heating through damping either by wave-particle interaction or by dissipation. However, most of the wave heating scenarios for the corona are based on the single-fluid theory of magnetohydrodynamics (MHD) and, hence, electrostatic drift waves have not been investigated in great detail, while the system itself is highly inhomogeneous. In almost all of the suggested wave mechanisms for coronal heating, the main energy source is located in other regions and is provided to the corona by means of waves carrying the energy through the lower atmospheric layers. Here, we point out an important energy source available within the corona itself to excite short-scale electrostatic perturbations, namely the many sheared plasma flows present in the corona. The mentioned electrostatic perturbations can be associated with either the "purely unstable mode" (D'Angelo 1965) or the drift waves (from kinetic theory or two-fluid plasma model) depending upon the scales of the density inhomogeneity and the wavelengths. Vranjes \& Poedts (2009a) have proposed a new paradigm of solar coronal heating by the latter drift waves. These authors have used results from kinetic theory which predicts that the drift wave is a universally unstable mode and the density gradient in the direction normal to the magnetic flux surfaces is the source of its instability, which is also the cause of its existence. It has been proposed that the drift waves can heat the corona through two possible ways, viz., due to Landau damping effects in the direction parallel to the magnetic field, and due to stochastic heating in the perpendicular direction. It has been confirmed (De Pontieu et al. 2007) that the solar atmosphere is highly structured and has inhomogeneous density filaments of various sizes. Therefore, the drift waves in the solar corona may have many different scales of wavelengths.

Vranjes \& Poedts (2009a) assumed that the external magnetic field is directed along the $z$-axis while the plasma density has 
a gradient along the $x$-axis and the wave propagates, under the local approximation, in the $y z$-plane with a perpendicular wavelength $\lambda_{y}=2 \pi / k_{y}$ and a parallel wavelength $\lambda_{z}=$ $2 \pi / k_{z}$, while $k_{z} \ll k_{y}$ (where $k_{y}$ and $k_{z}$ are the respective wave numbers). As an example, drift waves have been considered with $\lambda_{y} \simeq 0.5 \mathrm{~m}$ while $\lambda_{z}=20 \mathrm{~km}$ and $L_{n}=100 \mathrm{~m}$ (where $L_{n}=\left|\left(1 / n_{0}\right) d n_{0} / d x\right|^{-1} \mathrm{~m}$ ) is the inhomogeneity scale length, i.e., the density gradient scale length. Vranjes \& Poedts (2009a) demonstrated that heating can take place at various inhomogeneity scale lengths $L_{n}$ by scanning this parameter while keeping the ratio $\lambda_{z} / L_{n}$ fixed, e.g., setting $L_{n}=s \times 100 \mathrm{~m}$ and $\lambda_{z}=s \times 40000 \mathrm{~m}$ and varying the parameter $s$. These authors then showed that the ratio of the frequency and the growth rate, for the fixed value $\lambda_{y}=0.5 \mathrm{~m}$, is $\omega_{r} / \omega_{i} \simeq 1$ for $1 \leqslant s \leqslant 1000$. Note that the considered variation of the parameter $s$ demonstrates that heating occurs also everywhere along a given flux tube, i.e., when the radial density gradient varies with increased altitude.

We here focus our attention on the fact that also sheared flows, i.e., flows with an inhomogeneous velocity profile, are omnipresent in the corona. Thus, a large source of free energy for the excitation of short-scale electrostatic perturbations exists within the corona. Two types of electrostatic instabilities are expected to occur throughout the corona continuously.

1. The purely growing sheared flow-driven instability in the Doppler-shifted frame (D’Angelo 1965), which exists even if the plasma density is uniform.

2. The drift wave instability that needs a plasma density gradient for its existence (Kadomtsev \& Timofeev 1963) and sheared flows for instability (Saleem et al. 2007).

We here only investigate drift waves with relatively longer wavelengths compared to the ion Larmor radius, so that the use of the fluid model is justified. Moreover, the electrons are assumed to follow the Boltzmann distribution. Therefore, the frequency $\omega_{r}$ of the drift waves must fulfill the condition $\omega_{r} \ll v_{t e} k_{z}$, where $v_{t e}=\left(T_{e} / m_{e}\right)^{1 / 2}$ is the electron thermal velocity and $k_{z}$ is the wave number parallel to the external magnetic field. In general, the drift waves and the purely growing D'Angelo mode have shorter wavelengths compared to the wellknown Alfvén waves of the MHD model. The electrostatic perturbations driven by sheared flows can transfer their energy to plasma particles. For the case of drift waves, a detailed picture has been presented by Vranjes \& Poedts (2009b, 2009c).

The remainder of this paper is organized as follows. The theoretical three-fluid model is described in the following section where the equations are given first and then a fourthorder dispersion relation is derived. In Section 3, this model is applied to the solar corona, while in Section 4 the role of dissipation is discussed. Finally, in Section 5 we discuss the obtained results and their consequences for the problem of solar coronal heating.

\section{THEORETICAL MODEL}

Let us consider the collisionless coronal plasma consisting of electrons and two types of ions which are denoted with the subscript $j=a, b$, where " $a$ " represents hydrogen ions and " $b$ " corresponds to helium ions. We choose the $z$-axis in the direction of the uniform ambient magnetic field, such that $\mathbf{B}_{0}=B_{0} \hat{\mathbf{z}} \equiv$ constant. The inhomogeneity of the plasma density and the shear background flow (along the magnetic field) is chosen in the $x$-direction, i.e., $\nabla n_{j 0}=-\left|d n_{j 0} / d x\right| \hat{\mathbf{x}}$, and the shear flow then is given by $v_{j 0}(x) \hat{\mathbf{z}}=v_{0}(x) \hat{\mathbf{z}}$, where $v_{0}$ is the same for all species, i.e., for electrons and both types of ions. Each species has a zero-order diamagnetic drift $\mathbf{v}_{D j}=-\left(T_{j} / q_{j} B_{0}\right) \nabla \ln n_{j 0} \times \hat{\mathbf{z}}$, where $T_{j}$ is the temperature and $q_{j}$ denotes the charge of the $j$ th species of ions. For electrostatic perturbations, the momentum equation yields the perpendicular component of the velocity of $j$ th species of ions as

$$
\begin{aligned}
\mathbf{v}_{\perp j}= & \underbrace{\frac{1}{B_{0}}\left(\mathbf{E}_{\perp} \times \hat{\mathbf{z}}\right)}_{=\mathbf{v}_{E}} \underbrace{-\frac{T_{j}}{q_{j} B_{0}}\left(\nabla \ln n_{j} \times \hat{\mathbf{z}}\right)}_{=\mathbf{v}_{D j}} \underbrace{-\frac{1}{q_{j} B_{0}}\left(\frac{\nabla \cdot \boldsymbol{\Pi}_{j}}{n_{j}}\right)}_{=\mathbf{v}_{\Pi j}} \\
& \underbrace{-\frac{1}{\Omega_{j}}\left(\partial_{t}+\mathbf{v}_{j} \cdot \nabla\right) \mathbf{v}_{j} \times \hat{\mathbf{z}},}_{=\mathbf{v}_{p j}}
\end{aligned}
$$

where $\mathbf{v}_{E}, \mathbf{v}_{D j}, \mathbf{v}_{\Pi j}$, and $\mathbf{v}_{p j}$ are the electric, the diamagnetic, the stress tensor, and the polarization drifts, respectively. The parallel component of the linearized ion momentum equation(s) then gives

$$
\left(\partial_{t}+v_{j 0 z} \partial_{z}\right) v_{j z 1}+v_{j z 1} d_{x} v_{j 0 z}(x)=\frac{q_{j}}{m_{j}} E_{z 1}-\frac{T_{j 0}}{m_{j} n_{j 0}} \partial_{z} n_{j 1} .
$$

Here, we used the notation $d_{x}=d / d x$ and the subscripts zero (0) and one (1) denote zero-order and linear quantities, respectively. The continuity equation for the $j$ th ions is given by

$$
\begin{gathered}
\left(\partial_{t} n_{j 1}+v_{0 j z}\right) \partial_{t} n_{j 1}+\nabla n_{j 0} \cdot \mathbf{v}_{E}+\frac{n_{j 0}}{B_{0} \Omega_{j}}\left(\partial_{t}+v_{j 0 z} \partial_{z}\right) \nabla_{\perp} \cdot \mathbf{E}_{\perp} \\
-\frac{T_{j}}{q_{j} B_{0} \Omega_{j}}\left(\partial_{t}+v_{j 0 z} \partial_{z}\right) \nabla_{\perp}^{2} n_{j 1}+n_{j 0} \partial_{z} v_{j z 1}=0,
\end{gathered}
$$

where $\Omega_{j}=q_{j} B_{0} / m_{j}$ and $\nabla v_{0 z}(x)=+\left|d \nu_{0}(x) / d x\right| \hat{\mathbf{x}}$. For deriving Equation (3), we have used the following relation:

$$
\begin{gathered}
\nabla \cdot\left[n_{j} \cdot\left(\mathbf{v}_{j p}+\mathbf{v}_{j \pi}\right)\right]=\frac{n_{j 0}}{B_{0} \Omega_{j}} \partial_{t}\left(\nabla_{\perp} \cdot \mathbf{E}_{\perp 1}\right)-\frac{T_{j}}{q_{j} B_{0} \Omega_{j}} \partial_{t} \nabla^{2} n_{j 1} \\
+\frac{n_{j 0}}{\Omega_{j}} v_{0}(x) \partial_{z}\left\{\frac{1}{B_{0}} \nabla_{\perp} \cdot \mathbf{E}_{\perp}-\frac{T_{j}}{q_{j} B_{0}} \frac{\nabla_{\perp}^{2} n_{j 1}}{n_{j 0}}\right\} .
\end{gathered}
$$

The detailed derivation of Equation (4) is given in the second chapter of Weiland (2000) for $v_{0}=0$ and has been modified here due to the effects of the sheared flows.

The continuity equations for $j=a, b$ can also be expressed as

$$
R_{a}^{2} n_{a 1}=-n_{a 0} S_{a}^{2} \Phi_{1}
$$

and

$$
R_{b}^{2} n_{b 1}=-n_{b 0} S_{b}^{2} \Phi_{1}
$$

where

$$
\begin{gathered}
R_{a}^{2}=\left(1+\rho_{a T}^{2} k_{y}^{2}\right) \Omega_{\omega}^{2}-v_{t e}^{2} k_{y}^{2}, \\
R_{b}^{2}=\left(1+\rho_{b T}^{2} k_{y}^{2}\right) \Omega_{\omega}^{2}-v_{t e}^{2} k_{y}^{2}, \\
S_{a}^{2}=\left\{-\omega_{a}^{*} \Omega_{\omega}+\rho_{a s}^{2} k_{y}^{2} \Omega_{\omega}^{2}+c_{a s}^{2} k_{y} k_{z} \frac{1}{\Omega_{a}} \frac{d v_{0}}{d x}-c_{a s}^{2} k_{z}^{2}\right\}, \\
S_{b}^{2}=\left\{-\omega_{b}^{*} \Omega_{\omega}+\rho_{b s}^{2} k_{y}^{2} \Omega_{\omega}^{2}+c_{b s}^{2} k_{y} k_{z} \frac{1}{\Omega_{b}} \frac{d v_{0}}{d x}-c_{b s}^{2} k_{z}^{2}\right\} .
\end{gathered}
$$

Here, we have defined the following quantities: $v_{t j}^{2}=T_{j} / m_{j}$, $c_{j s}^{2}=T_{e} / m_{j}, \Omega_{\omega}=\left(\omega-\omega_{0}\right), \omega_{0}=v_{0} k_{z}, \omega_{j}^{*}=D_{e} \kappa_{n j} \kappa_{y}$, 
$D_{e}=T_{e} / e B_{0}, \kappa_{j n}=\left|\left(1 / n_{j 0}\right) d n_{j 0} / d x\right|, \Phi_{1}=e \varphi_{1} / T_{e}$, $\rho_{j s}=\left(T_{e} / m_{j} \Omega_{j}^{2}\right)^{1 / 2}$, and $\rho_{j T}=\left(T_{j} / m_{j} \Omega_{j}^{2}\right)^{1 / 2}$.

The Poisson equation in this case can be written as

$$
\nabla \cdot \mathbf{E}_{1}=\frac{e}{\epsilon_{0}}\left(n_{a 1}+n_{b 1}-n_{e 1}\right)
$$

The electrons are assumed to follow the Boltzmann relation

$$
n_{e 1} \simeq n_{e 0} e^{\Phi_{1}}
$$

In a steady state, the relation $n_{e 0}=n_{a 0}+n_{b 0}$ holds. Equations (5)-(8) yield a fourth-order dispersion relation as follows:

$$
L_{4} \Omega_{\omega}^{4}+L_{3} \Omega_{\omega}^{3}+L_{2} \Omega_{\omega}^{2}+L_{1} \Omega_{\omega}+L_{0}=0
$$

where

$$
\begin{aligned}
L_{4}=\Lambda^{2} \alpha_{a}^{2} \alpha_{b}^{2}+ & \frac{n_{a 0}}{n_{e 0}}\left(\alpha_{b}^{2} \rho_{a s}^{2} k_{y}^{2}\right)+\frac{n_{b 0}}{n_{e 0}}\left(\alpha_{a}^{2} \rho_{b s}^{2} k_{y}^{2}\right), \\
L_{3}= & -\left\{\frac{n_{a 0}}{n_{e 0}} \alpha_{b}^{2} \omega_{a}^{*}+\frac{n_{b 0}}{n_{e 0}} \alpha_{a}^{2} \omega_{b}^{*}\right\}, \\
L_{2}= & -\Lambda^{2}\left(\alpha_{a}^{2} v_{b T}^{2} k_{z}^{2}+\alpha_{b}^{2} v_{a T}^{2} k_{z}^{2}\right) \\
& +\frac{n_{a 0}}{n_{e 0}}\left(\alpha_{b}^{2} g_{a} c_{a s}^{2} k_{z}^{2}-\rho_{a s}^{2} k_{y}^{2} v_{b T}^{2} k_{z}^{2}\right) \\
& +\frac{n_{b 0}}{n_{e 0}}\left(\alpha_{a}^{2} g_{b} c_{b s}^{2} k_{z}^{2}-\rho_{b s}^{2} k_{y}^{2} v_{a T}^{2} k_{z}^{2}\right), \\
L_{1}= & \frac{n_{a 0}}{n_{e 0}}\left(v_{b T}^{2} k_{z}^{2} \omega_{a}^{*}\right)+\frac{n_{b 0}}{n_{e 0}}\left(v_{a T}^{2} k_{z}^{2} \omega_{b}^{*}\right),
\end{aligned}
$$

and

$$
L_{0}=\Lambda^{2} v_{a T}^{2} k_{z}^{2} v_{b T}^{2} k_{z}^{2}-\frac{n_{a 0}}{n_{e 0}} g_{a} v_{b T}^{2} k_{z}^{2} c_{a s}^{2} k_{z}^{2}-\frac{n_{b 0}}{n_{e 0}} g_{b} v_{a T}^{2} k_{z}^{2} c_{b s}^{2} k_{z}^{2} .
$$

Here, $g_{j}=\left(\frac{k_{y}}{k_{z}} A_{j}-1\right), A_{j}=\frac{1}{\Omega_{j}} \frac{d v_{0}}{d x}, \alpha_{j}^{2}=\left(1+\rho_{j T}^{2} k_{y}^{2}\right)$, $\Lambda^{2}=\left(1+\lambda_{D e}^{2} k^{2}\right)$, and $\lambda_{D e}=\left(\epsilon_{0} T_{e} / n_{e 0} e^{2}\right)^{1 / 2}$.

\section{APPLICATION TO THE SOLAR CORONA}

In this section, we will show that the theoretical model presented above is perfectly applicable to solar coronal plasma. Let us choose the parameters of the corona (Priest 1982) as $n_{e 0}=10^{15} \mathrm{~m}^{-3}, n_{a 0}=0.9 n_{e 0}, n_{e 0}=0.1 n_{e 0}$, and $T_{e}=10^{6} \mathrm{~K}$. Since the condition $T_{e}<T_{\mathrm{H}}<T_{\mathrm{He}}$ holds in this plasma (Hansteen et al. 1997), we assume $T_{a}=2.5 T_{e}$ and $T_{b}=3 T_{e}$.

First we shall show that the sheared flows of electron-proton plasma of corona give rise to both purely growing and oscillatory drift wave instabilities. Then it will be shown that the presence of the second ion (helium, with an abundance of 10\%) in this plasma modifies the growth rates and the real frequencies of the drift waves in different parameter regimes. If this $10 \%$ concentration of the ions in the plasma is neglected, then we may use $n_{b 0}=0$ and $T_{b}=0$ in Equation (9). In the limit $\lambda_{D e}^{2} k^{2} \ll 1$, we have $\Lambda^{2}=1, \alpha_{b}^{2}=1, n_{a 0} / n_{e 0}=1, v_{b T}^{2}=0$ and it gives $L_{4}=\rho_{a s}^{2} k_{y}^{2}+\alpha_{a}^{2}, L_{3}=-\omega_{a}^{*}, L_{2}=-v_{a T}^{2} k_{z}^{2}+g_{a}$ $c_{a s}^{2} k_{z}^{2}$, and $L_{1}=0=L_{0}$. Hence, Equation (9) reduces to

$$
\Lambda_{0} \Omega_{\omega}^{2}-\omega_{a}^{*} \Omega_{\omega}+k_{z} k_{y} c_{a s}^{2}\left\{A_{a}-\left(1+v_{a T}^{2} / c_{a s}^{2}\right) k_{z} / k_{y}\right\}=0
$$

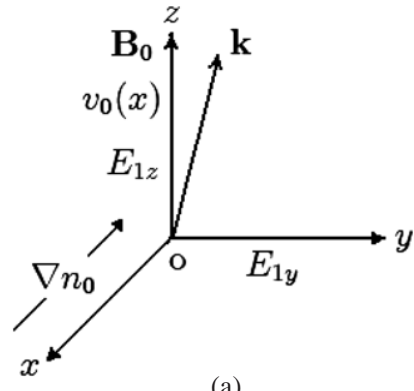

(a)

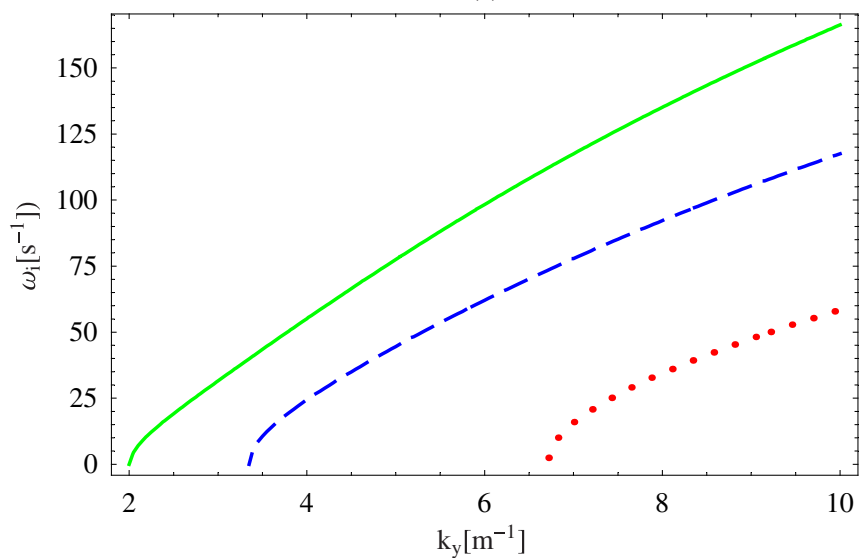

(b)

Figure 1. (a) Directions of shear flow $v_{0}(x)$ and the wave propagation with respect to density gradient and the external magnetic field are shown. (b) The growth rate $\left(\omega_{i}\right)$ of the sheared flow-driven instability is plotted against the perpendicular component of the wave number $\left(k_{y}\right)$ for different inverse velocity scale lengths, viz., $\kappa_{v}=k_{y} / 60$ (solid curve), $\kappa_{v}=k_{y} / 100$ (dashed curve), and $\kappa_{v}=k_{y} / 200$ (dotted curve), for $n_{e 0} \sim n_{a 0} \sim 10^{15} \mathrm{~m}^{-3}, T_{e}=10^{6} \mathrm{~K}$, $T_{a}=2.5 T_{e}, B_{0} \sim 10^{-2} \mathrm{~T}$, and $v_{0}=10 \mathrm{~km} \mathrm{~s}^{-1}$, using $\kappa_{n}=0, n_{b 0}=0$, and $k_{z}=10^{-4} k_{y}$.

(A color version of this figure is available in the online journal.)

This quadratic equation has two roots, viz.,

$$
\begin{aligned}
\left(\Omega_{\omega}\right)_{1,2}= & \frac{1}{2 \Lambda_{0}}\left\{\omega_{a}^{*} \pm\left[\left(-\omega_{a}^{*}\right)^{2}+4 \Lambda_{0} k_{z} k_{y} c_{a s}^{2}\right.\right. \\
& \left.\left.\times\left(\left(1+\sigma_{a}\right) \frac{k_{z}}{k_{y}}-A_{a}\right)\right]^{1 / 2}\right\},
\end{aligned}
$$

where $\Lambda_{0}=\left(1+\rho_{a T}^{2} k_{y}^{2}+\rho_{a s}^{2} k_{y}^{2}\right)$ and $\sigma_{a}\left(=T_{a} / T_{e}\right)$. Thus, the following conditions should be satisfied simultaneously for the instability to occur:

$$
\left(1+\sigma_{a}\right) \frac{k_{z}}{k_{y}}<A_{a}
$$

and

$$
\left(\omega_{a}^{*}\right)^{2}<4 \Lambda_{0} c_{a s}^{2} k_{z} k_{y}\left|\left(\left(1+\sigma_{a}\right) \frac{k_{z}}{k_{y}}-A_{a}\right)\right| .
$$

We shall assume that $L_{j n}=1 /\left|d \ln n_{j 0} / d x\right|$, i.e., the inhomogeneity scale length has the same value for all species so that it can be denoted by $L_{n}$.

First, we discuss the electrostatic instabilities mentioned above under coronal circumstances but ignore the presence of the $10 \%$ helium abundance. Figure 1(a) shows just the wave propagation with respect to the external magnetic field and density gradient. In Figure 1(b), the growth rates of the shear flow-driven electrostatic instability are plotted (using 

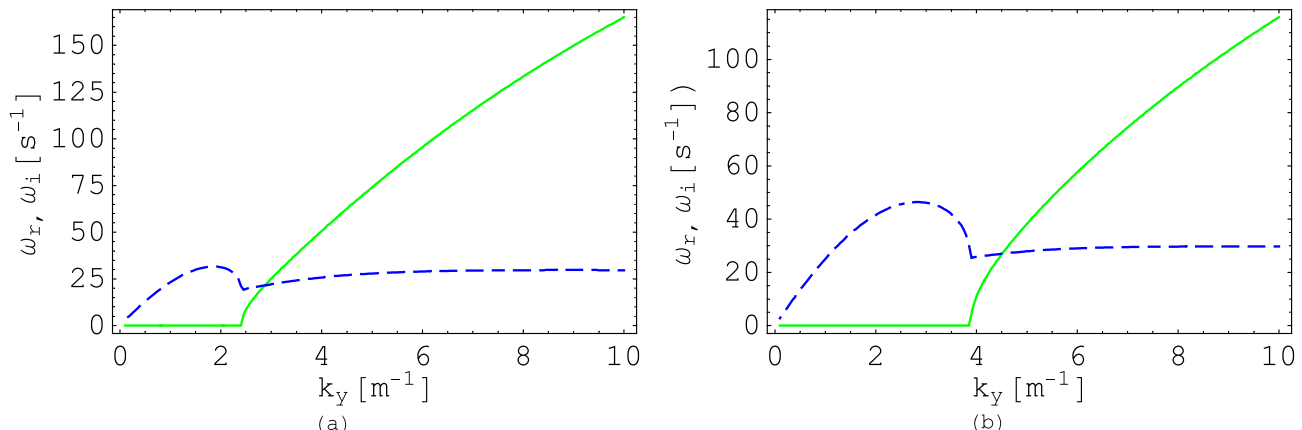

Figure 2. Real and imaginary parts of the frequencies of the perturbations ( $\omega_{r}$ (dashed curves) and $\omega_{i}$ (solid curves)) are plotted against the perpendicular component of the wave number $\left(k_{y}\right)$ for varying inverse velocity scale lengths (a) $\kappa_{v}=k_{y} / 60$ and (b) $\kappa_{v}=k_{y} / 100$, taking $v_{0}=10 \mathrm{~km} \mathrm{~s}^{-1}, \kappa_{n}=1.9 \times 10^{-3} \mathrm{~m}^{-1}, n_{b 0}=0$, and $k_{z}=10^{-4} k_{y}$. All other parameter values are the same as in Figure 1.

(A color version of this figure is available in the online journal.)
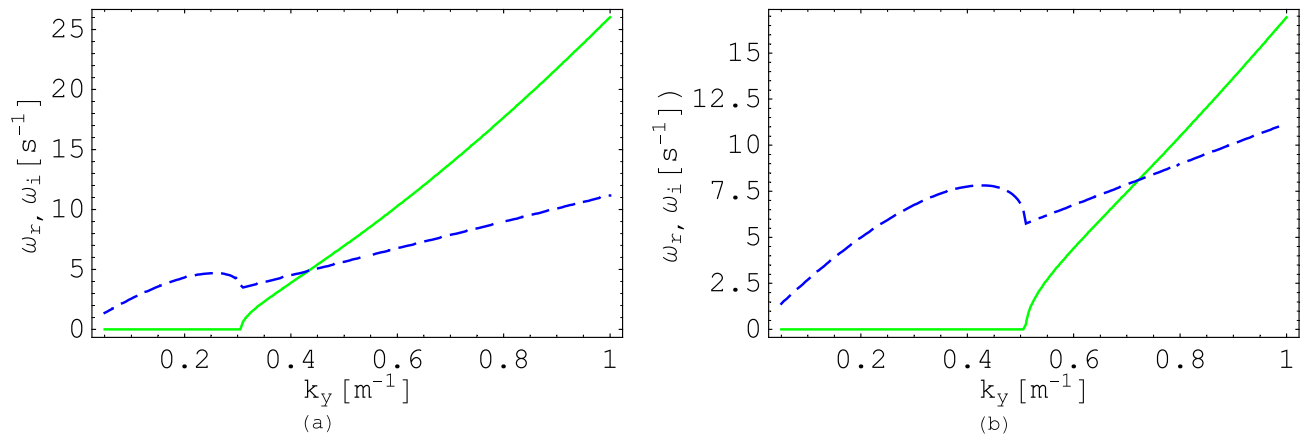

Figure 3. Real and imaginary parts of the frequencies of the electrostatic perturbations ( $\omega_{r}$ (dashed curves), $\omega_{i}$ (solid curves)) are plotted against the perpendicular component of the wave number $\left(k_{y}\right)$ for changing inverse velocity scale lengths (a) $\kappa_{v}=k_{y} / 60$ and (b) $\kappa_{v}=k_{y} / 100$, for $v_{0}=70 \mathrm{~km} \mathrm{~s}^{-1}, \kappa_{n}=1.9 \times 10^{-3} \mathrm{~m}^{-1}$, $n_{b 0}=0$, and $k_{z}=10^{-4} k_{y}$. All other parameters have the same values as in Figure 1. Since $k_{y}$ is 10 times smaller than the values of Figure 2, therefore both real and imaginary frequencies turn out to be smaller in this case although $v_{0}$ is seven times larger.

(A color version of this figure is available in the online journal.)

Equation (10)) against the perpendicular component of the wave number $\left(k_{y}\right)$ for different gradient scale lengths of the plasma flow parallel to the external magnetic field, in the case of a homogeneous plasma density, i.e., $\kappa_{n}=0$. The solid curve corresponds to an inverse velocity scale length $\kappa_{v}=k_{y} / 60$, while the dashed curve corresponds to $\kappa_{v}=k_{y} / 100$, and the dotted curve to $\kappa_{v}=k_{y} / 200$. The other parameter values are given in the figure caption. The real frequency in the laboratory frame is $\omega_{0}=v_{0} k_{z}$. Since $\Omega_{\omega}=\omega-\omega_{0}$, and we assume $\omega=\omega_{r} \pm i \omega_{i}$, therefore, in the case of homogeneous $\left(\kappa_{n}=0\right)$ plasma, $\Omega_{\omega}= \pm i \omega_{i}$, where $\omega_{i}$ is real. The Doppler-shifted frequency of the purely growing mode is $\omega_{i}$ and is plotted versus the perpendicular wave number $k_{y}$. We note that the frequency $\omega_{i}$ decreases corresponding to the same $k_{y}$ when the shear flow gradient scale length $L_{v}=1 / \kappa_{v}$ increases. Thus, steeper gradients in the velocity shear profile give rise to larger growth rates of the instability.

In the presence of a plasma density gradient, i.e., $\kappa_{n} \neq 0$, the electrostatic drift wave can also be excited. For $v_{0}=0$ and $c_{a s}^{2} k_{z} k_{y} \ll\left(\omega_{a}^{*}\right)^{2}$, Equation (11) yields a stable drift wave with oscillation frequency $\omega_{r}^{*}=\omega_{a}^{*} / \Lambda_{0}$. If conditions (12) and (13) are satisfied, then $\left(\Omega_{\omega}\right)_{1,2}=\left(1 / 2 \Lambda_{0}\right)\left(\omega_{a}^{*} \pm i \gamma\right)$, where $\gamma$ is real with $0<\gamma$. If $\omega$ is the frequency of the perturbation then $\omega=\omega_{r} \pm i \omega_{i}$ and we have $\omega_{r}=\left(\omega_{a}^{*} / 2 \Lambda_{0}+\omega_{0}\right)$, while $\omega_{i}=\gamma / 2 \Lambda_{0}$. In Figure 2, the real and imaginary parts of the electrostatic perturbations ( $\omega_{r}$ (dashed curves) and $\omega_{i}$ (solid curves)) are plotted against the perpendicular component of the wave number $\left(k_{y}\right)$ for varying inverse velocity scale lengths, viz., (a) $\kappa_{v}=k_{y} / 60$ and (b) $\kappa_{v}=k_{y} / 100$, taking $v_{0}=10 \mathrm{~km} \mathrm{~s}^{-1}$, $\kappa_{n}=1.9 \times 10^{-3} \mathrm{~m}^{-1}, n_{b 0}=0$, and $k_{z}=10^{-4} k_{y}$; while all other parameter values are the same as in Figure 1. Figure 2 shows that the drift wave becomes unstable for a very short range of wavelengths $2<k_{y}<2.5$. Somewhere in between $k_{y}=2$ and $k_{y}=2.5$, the real frequency of the drift wave $\omega_{r}^{*}$ is larger than the growth rate $\omega_{i}$, that is, $\omega_{i}<\omega_{r}^{*}$ and, hence, linear analysis is valid. Note that the drift wave is driven by the sheared flow in this case. In Figure 2(a), assuming $\kappa_{v}=k_{y} / 60$, the purely growing instability dominates for $2.5 \leqslant k_{y}$ because here $\omega_{r} \ll \omega_{i}$ and hence it shows the shear flow-driven instability with local real frequency $\omega_{r}=\omega_{r}^{*}-\omega_{0}$. For steeper sheared flow gradient, the drift wave instability occurs at relatively larger values of $k_{y}$ as shown in Figure 2(b). The drift wave and D'Angelo's mode are mixed due to $\kappa_{n} \neq 0$ for $2<k_{y}<2.5$. But the instability for $2.5<k_{y}$ is basically the sheared flow-driven instability because linear theory for drift waves does not remain valid in the regime where $\omega_{r} \lesssim \omega_{i}$. In Figure 3, the real and imaginary parts of the frequencies of the mixed modes ( $\omega_{r}$ (dashed curves), $\omega_{i}$ (solid curves)) are plotted against the perpendicular component of the wave number $\left(k_{y}\right)$ for changing inverse velocity scale lengths (a) $\kappa_{v}=k_{y} / 60$ and (b) $\kappa_{v}=k_{y} / 100$, for $v_{0}=70 \mathrm{~km} \mathrm{~s}^{-1}$, $\kappa_{n}=1.9 \times 10^{-3} \mathrm{~m}^{-1}, n_{b 0}=0$, and $k_{z}=10^{-4} k_{y}$. All other parameters are the same as in Figure 1. Note that the growth rates and the real parts of the frequencies of the mixed modes both decrease corresponding also to smaller $k_{y}$ and $k_{z}$ compared to 

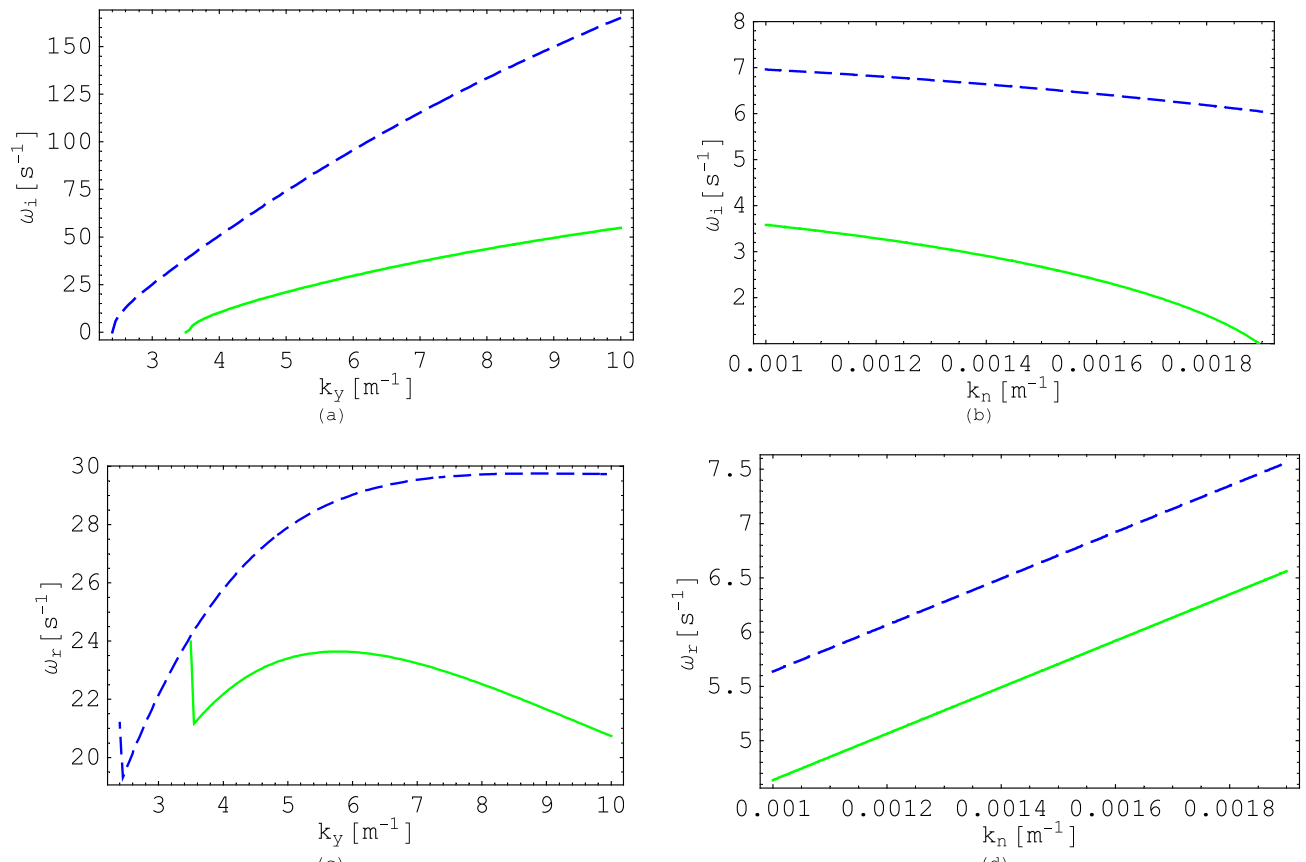

Figure 4. Growth rates $\left(\omega_{i}\right)$ of the perturbations are plotted against the perpendicular component of the wave number $\left(k_{y}\right)$ and the inverse density scale length $\left(\kappa_{n}\right)$, respectively, for a varying parallel component of the wave number, viz., (a) $k_{z}=10^{-5} k_{y}$ (solid curve) and $k_{z}=10^{-4} k_{y}$ (dashed curve) with fixed values of $v_{0}=10 \mathrm{~km} \mathrm{~s}^{-1}, \kappa_{n}=1.9 \times 10^{-3} \mathrm{~m}^{-1}$, and $\kappa_{v}=k_{y} / 60$, and corresponding to higher streaming velocities (b) $v_{0}=50 \mathrm{~km} \mathrm{~s}^{-1}$ (solid curve) and $v_{0}=70 \mathrm{~km} \mathrm{~s}^{-1}$ (dashed curve), for $\kappa_{v}=k_{y} / 60, k_{y}=0.5 \mathrm{~m}^{-1}$, and $k_{z}=10^{-4} k_{y}$. All other parameters are the same as in Figure 1 . The variations of the real frequency $\left(\omega_{r}\right)$ of the perturbations are also plotted against $k_{y}$ and $\kappa_{n}$, respectively, as shown in panels (c) and (d).

(A color version of this figure is available in the online journal.)

Figure 2, which is quite natural. In this case, the instabilities require larger values of flow $v_{0}(x)$ in order to satisfy condition (12).

The effect of the ratio of $k_{z} / k_{y}$ and the different streaming velocities $v_{0}$ on instabilities is shown in Figure 4. Here, the growth rate $\left(\omega_{i}\right)$ of the electrostatic perturbations are plotted against the perpendicular component of the wave number $\left(k_{y}\right)$ and the inverse density scale length $\left(\kappa_{n}\right)$, respectively, for a varying parallel component of the wave number, viz., (a) $k_{z}=10^{-5} k_{y}$ (solid curve) and $k_{z}=10^{-4} k_{y}$ (dashed curve) with fixed values of $v_{0}=10 \mathrm{~km} \mathrm{~s}^{-1}, \kappa_{n}=1.9 \times 10^{-3} \mathrm{~m}^{-1}$, and $\kappa_{v}=k_{y} / 60$, and corresponding to different streaming velocities (b) $v_{0}=50 \mathrm{~km} \mathrm{~s}^{-1}$ (solid curve) and $v_{0}=70 \mathrm{~km} \mathrm{~s}^{-1}$ (dashed curve) for $\kappa_{v}=k_{y} / 60, k_{y}=0.5 \mathrm{~m}^{-1}$, and $k_{z}=10^{-4} k_{y}$. The real frequencies are also plotted corresponding to the above-mentioned values of $\kappa_{v}, k_{y}$, and $\kappa_{n}$, respectively, for (c) $k_{z}=10^{-5} k_{y}$ (solid curve) and $k_{z}=10^{-4} k_{y}$ (dashed curve) with fixed $v_{0}=10 \mathrm{~km} \mathrm{~s}^{-1}$ and (d) $v_{0}=50 \mathrm{~km} \mathrm{~s}^{-1}$ (solid curve) and $v_{0}=70 \mathrm{~km} \mathrm{~s}^{-1}$ (dashed curve) for $k_{y}=0.5 \mathrm{~m}^{-1}$. All other parameters have the same values as in Figure 1. Since the chosen value of $k_{y}$ is smaller, the instability occurs at larger values of the streaming velocity. Both $\omega_{a}^{*}$ and $c_{a s}^{2} k_{z} k_{y}$ then become smaller and, therefore, we note that $\omega_{i}$ in Figure 4(b) and $\omega_{r}$ in Figure 4(d) turn out to be smaller than that shown in Figure 2 where $k_{y}$ is larger.

It is pointed out that the presence of $10 \%$ helium ions in the corona has a significant effect on the imaginary and real parts of the frequency of the electrostatic perturbations driven by the sheared flow. In Figure 5, the real and imaginary parts of the frequencies $\omega_{r}$ and $\omega_{i}$ are plotted against the perpendicular component of the wave number $\left(k_{y}\right)$, respectively, for $n_{b 0}=0$ (dashed curve) and $n_{b 0}=0.1 n_{e 0}$ (solid curve) corresponding to $\kappa_{n}=0$ (Figures $5(\mathrm{a})$ and (b)), and $\kappa_{n}=1.9 \times 10^{-3} \mathrm{~m}^{-1}$
(Figures 5(c) and (d)). Figure 5 thus displays the effect of the presence of helium ions on the real and imaginary parts of the frequencies due to the shear flow-driven instability (for $\kappa_{n}=0$ ) and the drift wave instability (for $\kappa_{n} \neq 0$ ). It is interesting to note that the purely growing D'Angelo's mode develops a real part of the frequency in a two-ion component hot plasma. The reason is that for $\nabla n_{a 0}=0$ and $\nabla n_{b 0}=0$, we have $L_{1}=L_{2}=0$ and Equation (9) becomes

$$
L_{4} \Omega_{\omega}^{4}+L_{2} \Omega_{\omega}^{2}+L_{0}=0 .
$$

This equation has complex roots corresponding to our range of parameters. The D'Angelo's modes for ions $a$ and $b$ couple together and develop a real part of the frequency. Substituting $n_{b 0}=0$ and $T_{b 0}=0$, we have $L_{0}=0$ and Equation (14) will give a Doppler-shifted purely growing instability as discussed in Figure 1. On the other hand, in the case of a non-uniform density, Figures 5(c) and (d) for $2<k_{y}<2.5$ show that $\omega_{i}<\omega_{r}^{*}$ and hence the linear theory for drift waves remains valid while the condition $\omega_{r}^{*} \ll v_{t e} k_{z}$ holds as well. Thus, we conclude that in the presence of helium ions and shear flows the drift waves are almost omnipresent in the corona to accelerate plasma particles. The large-amplitude drift waves will become nonlinear and this study is out of the scope of the present work.

\section{THE ROLE OF DISSIPATION}

The solar corona is commonly assumed to be a collisionless plasma because most of the wave studies deal with the relatively high-frequency Alfvén waves using the MHD equations or ion acoustic waves with wave numbers larger than our regime of parameters and, hence, the frequency becomes larger than the electron-ion collision frequency $v_{e i}$. The drift waves in the solar corona have been investigated for the first time (to the 

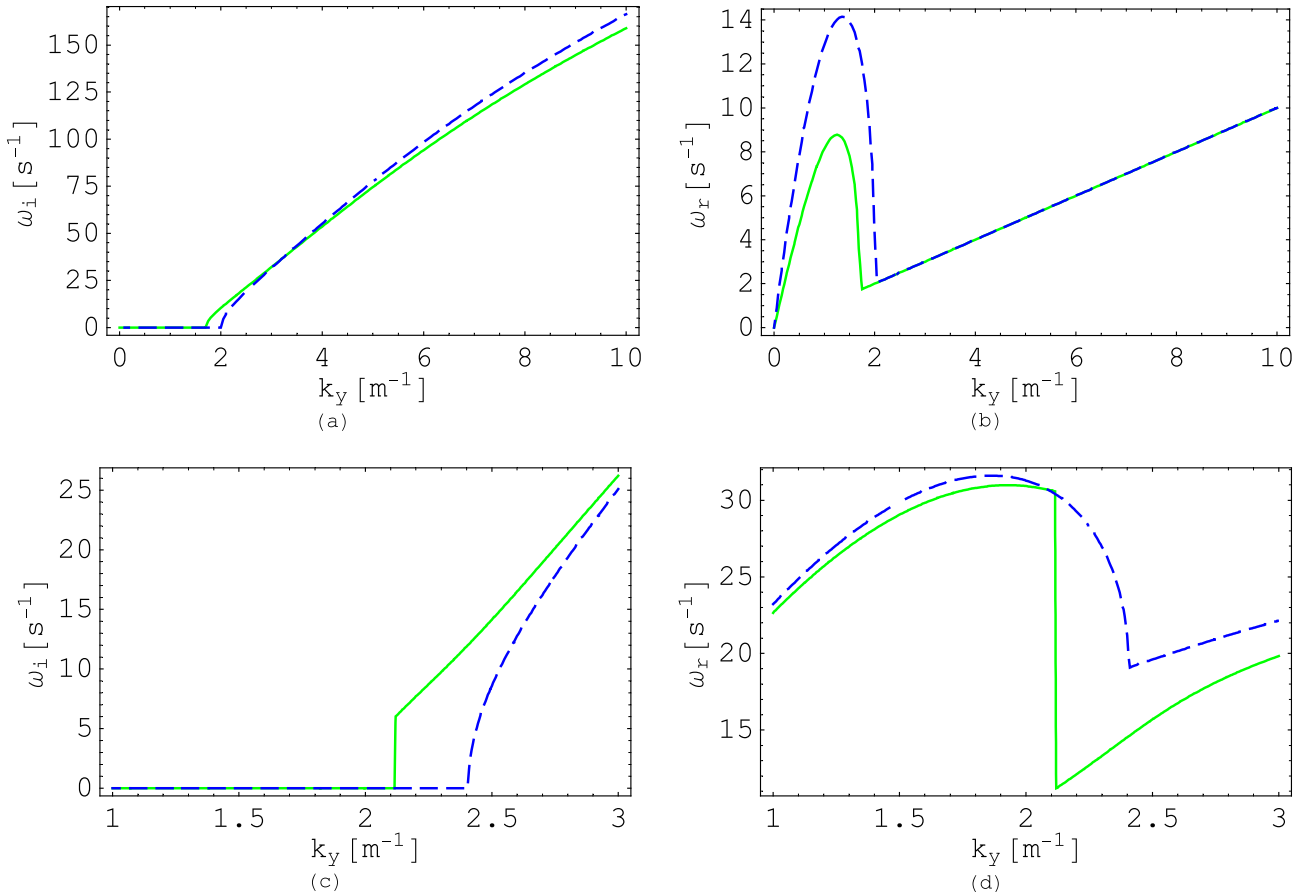

Figure 5. Real and imaginary parts of the frequencies of the perturbations $\left(\omega_{i}\right.$ and $\left.\omega_{r}\right)$ in the presence of sheared flow are plotted against the perpendicular component of the wave number $\left(k_{y}\right)$, respectively, for $n_{b 0}=0$ (dashed curve) and $n_{b 0}=0.1 n_{e 0}$ (solid curve) for $\kappa_{n}=0\left((\mathrm{a})\right.$ and (b)) and for $\kappa_{n}=1.9 \times 10^{-3} \mathrm{~m}^{-1}((\mathrm{c})$ and (d)). All other parameters have the same values as in Figure 1.

(A color version of this figure is available in the online journal.)

best of the authors' knowledge) by Vranjes \& Poedts (2009a, 2009b, 2009c). The drift waves investigated by Vranjes \& Poedts (2009a) have frequencies $\omega_{r} \sim \omega_{i} \sim 10^{2} \mathrm{rad} \mathrm{s}^{-1}$ while $v_{e i} \simeq 30$ $\mathrm{rad} \mathrm{s}^{-1}$ (Vranjes \& Poedts 2009b). Therefore, the ideal plasma approximation is justified. In the higher frequency $\left(\omega_{a}^{*}\right)$ regime, however, it does not seem preferable to assume the electrons to be inertialess because the condition $\omega_{a}^{*} \ll v_{t e} k_{z}$ may not remain satisfied.

We here chose the wave parameters such that the condition $\omega_{a}^{*}, \omega_{b}^{*} \ll v_{t e} k_{z}$ remains satisfied and the electrons follow the Boltzmann distribution. But in this regime, we see another small effect, namely the dissipation. Let us look back at Figure 2(a) to analyze the role of drift waves in corona. This wave is stable for $k_{y}<2$ and then for $2<k_{y}<2.5$, the wave develops $\omega_{i}$ and, for $2.5<k_{y}$ we can see that $\omega_{r}<\omega_{i}$ and the shear flow-driven purely growing instability dominates. To understand the wave behavior, we choose a value of $k_{y}$ in between 2 and 2.5. Let $k_{y}=2.2 \mathrm{~m}^{-1}$. Then for $\kappa_{n}=1.9 \times 10^{-3} \mathrm{~m}^{-1}$, we find $\omega_{a}^{*}=D_{e} \kappa_{n} k_{y} \simeq 36 \mathrm{rad} \mathrm{s}^{-1}$, which is nearly equal to $v_{e i}$. Therefore, dissipation can play some role in this frequency regime.

It is important, however, to note that the well-known drift dissipative instability (DDI; Weiland 2000) does not become important even in this range of frequencies because $\omega_{a}^{*}$ is not much smaller than $v_{e i}$. To obtain DDI, we use the parallel momentum equation for electrons (Weiland 2000),

$$
\frac{n_{e 1}}{n_{e 0}} \simeq \frac{e \varphi}{T_{e}}\left\{1-i \frac{v_{e i}}{v_{t e}^{2} k_{z}^{2}}\left(\omega_{a}^{*}-\omega\right)\right\}
$$

in the limit $\omega_{a}^{*} \ll v_{e i}$. Then the ion continuity equation, in the limit $c_{s}^{2} k_{z}^{2} \ll\left(\omega_{a}^{*}\right)^{2}$ along with quasi-neutrality $\left(n_{e} \simeq n_{i}\right)$, yields the linear dispersion relation for the DDI with the real frequency $\omega_{r d}$ and imaginary frequency $\omega_{i d}$ as (Weiland 2000)

$$
\omega_{r d} \simeq \frac{\omega_{a}^{*}}{\left(1+\rho_{a s}^{2} k_{y}^{2}\right)}
$$

and

$$
\omega_{i d} \simeq\left(\frac{\omega_{r}^{2}}{v_{t e}^{2} k_{z}^{2}}\right) v_{e i} \rho_{a s}^{2} k_{y}^{2},
$$

respectively. In our case, the drift wave frequency can be of the order of $v_{e i}$. We have shown that the drift wave becomes unstable due to the sheared flow in the absence of electron-ion collisions. Therefore, in our parameter regime, the dissipation can just add its small effect to the already unstable perturbation. However, the DDI is not applicable since the real frequency $\omega_{a}^{*}$ is not much smaller than $v_{e i}$. Now let us investigate the DDI in the solar corona as a matter of general interest.

Figure 2(a) indicates that for small $k_{y}$ values, the drift wave is stable and shear flow does not have an effect on it. Therefore, we expect that for longer wavelengths $k_{y} \ll 2$ the DDI may take place in the corona. As an example, let us choose $k_{y}=0.1 \mathrm{~m}^{-1}$ and take the rest of the parameter values to be the same, i.e., $\kappa_{n}=1.9 \times 10^{-3}, k_{z}=10^{-4} k_{y}, B_{0}=10^{-2} \mathrm{~T}$, etc. Then, we find $\omega_{a}^{*} \simeq 1.63, v_{t e} k_{z} \simeq 36$ while $v_{e i} \simeq 36$. Thus, $\omega_{a}^{*} \ll v_{e i} \ll \Omega_{i}$ holds along with $\omega_{a}^{*} \ll v_{t e}^{2} k_{z}^{2} / v_{e i}$. Therefore, in the corona the DDI gives rise to drift waves having very low frequency $\omega_{a}^{*} \simeq 1 \mathrm{~Hz}$ and relatively longer wavelength $\lambda_{y} \simeq 2 \pi / k_{y} \simeq 60 \mathrm{~m}$.

\section{DISCUSSION}

It has been proposed that perturbed electrostatic fields are generated throughout the solar corona, due to localized sheared plasma flows, which accelerate the particles and heat them. The corona is not a static spherical shell of plasma; rather, it 
has flows and is very inhomogeneous. Therefore, it has already been proposed that the free energy available in the form of density gradients can produce electrostatic drift waves (Vranjes \& Poedts 2009a, 2009b, 2009c). Using the results of kinetic theory, these authors have shown that the universally unstable drift waves can heat the coronal ions very efficiently. The density gradient in the direction perpendicular to the external magnetic field is the cause of the existence of these waves and is the source for their instability as well. The waves transfer their energy to plasma particles through Landau damping (the wave-particle interaction). This process cannot be studied using fluid models.

However, the two-fluid theory has predicted a KelvinHelmholtz-type instability which takes place in plasmas because of sheared flows (D'Angelo 1965). It shows that, if both electrons and ions flow with the same velocity along the external magnetic field $\mathbf{B}_{0}$, and there exists a gradient in the flow velocity in the direction perpendicular to $\mathbf{B}_{0}$, then the perturbed electrostatic field becomes unstable. These are purely growing fields in the frame of reference of the flow with a wide spectrum of wavelengths. But in the laboratory frame, these unstable perturbations have associated local real frequencies as $\omega_{0}=v_{0}(x) k_{z}$. The sheared flows can give rise to two types of electrostatic instabilities continuously throughout the corona.

1. The sheared flow-driven instability (D'Angelo 1965), even in regions where the plasma density is uniform $\left(\kappa_{n}=0\right)$.

2. The drift waves, which are excited by the sheared flows in the coronal regions where density is non-uniform.

The basic electrostatic drift wave is a stable low-frequency mode of an inhomogeneous plasma. In the presence of electron-ion collisions the plasma density and the electrostatic potential do not remain in phase as is clear from Equation (15), which is the same as Equation (3.18) of Weiland (2000). Similarly in the case of a sheared flow, a phase difference is produced between the parallel ion velocity and the electrostatic potential, which allows the wave to grow, which is clear from our Equation (2). The geometry of the perturbation is illustrated in Figure 1(a). The wave propagates obliquely, making a small angle with the $z$-axis in the $y z$-plane, perpendicular to the direction of density gradient. The plasma flows along the external magnetic field without creating a shear in the external magnetic field.

It is well known that D'Angelo's mode is purely growing in a single ion plasma. But we point out that in a two-ion plasma, this mode develops a small component of real frequency as well. Figure 5(b) clearly shows that $\omega_{r} \neq 0$ for the case $\kappa_{n}=0$ when $n_{b 0} \neq 0$. These instabilities have been investigated neglecting the effects of electron-ion collisions. For $k_{y} \simeq 10 \mathrm{~m}^{-1}$, we find that the sheared flow-driven instability can create potential $\varphi_{1} \simeq 68$ volts in about a growth time $\tau_{g} \simeq 0.03 \mathrm{~s}$ if at $t=0$ we assume $e \varphi_{1} / T_{e} \simeq 10^{-2}$. Note that Vranjes \& Poedts (2009a) have estimated that the large frequency drift waves $\left(\omega_{r} \simeq 2.5 \times 10^{2}\right)$ can give rise to this value of potential in $0.02 \mathrm{~s}$. The components of the wavelength chosen in the perpendicular and parallel directions are, respectively, $\lambda_{y}=0.5 \mathrm{~m}$ and $\lambda_{z}=20 \mathrm{~km}$. Then they have $v_{t e} k_{z}=1.2 \times 10^{3} \mathrm{rad} \mathrm{s}^{-1}$.

Figure 2 shows that the drift waves having $\omega_{r}<50 \mathrm{rad} \mathrm{s}^{-1}$ satisfying $\omega_{r} \ll v_{t e} k_{z}$ can become unstable due to sheared flow. The collisional effects have been neglected which can only modify the growth rates by small amounts in this parameter regime. It is well known that the electron-ion collisions can drive the DDI and does not require plasma flow to occur. The collisions produce electrostatic drift waves having very small real frequency $\omega_{r d} \simeq 1.6 \mathrm{rad} \mathrm{s}^{-1}$, corresponding to $k_{y} \simeq 0.1 \mathrm{~m}^{-1}$ and longer wavelengths $\lambda_{y}=2 \pi / k_{y} \simeq 60 \mathrm{~m}$. If the initial perturbation is assumed to be $e \varphi_{1} / T_{e}=10^{-2}$, then $\varphi_{1}$ will take time $\tau_{g} \simeq 15$ minutes to increase up to $\varphi_{1}=86$ volts. But smaller values of $\varphi_{1}$ will be produced on much smaller timescales than 15 minutes.

Our estimates show that the electrostatic instabilities driven by the sheared flows and dissipation continue growing linearly during the time denoted by growth time $\tau_{g}$. The electrostatic potentials can attain about 100 volts in $\tau_{g}=0.03 \mathrm{~s}$ in the case of a sheared flow-driven instability, and in $\tau_{g}=15$ minutes, in the case of a DDI, if the normalized initial potential perturbation is assumed to be $1 \%$. However, the nonlinearities may saturate the growth of these instabilities before time $\tau_{g}$.

Thus, we conclude that drift waves of different frequencies and wavelengths are produced in the solar corona due to sheared flows and electron-ion collisions. As a result, electrostatic fields are almost omnipresent in the coronal plasma and continuous self-heating is taking place due to the sheared plasma flows. The present investigation thus shows that even if the plasma density is uniform in a region, the electrostatic fields will still be produced because the corona is not static and sheared flows occur everywhere. The purely growing D'Angelo's mode also develops a small real part of the frequency because the solar corona contains two types of ions. Therefore, we propose that the sheared flow-driven instabilities play an important role in the self-heating of the corona.

These results were obtained in the framework of projects GOA/2009-009 (K. U. Leuven), G.0729.11 (FWOVlaanderen), and C 90347 (ESA Prodex 9). Financial support by the European Commission through SOTERIA (Collaborative project 218816 of FP7-SPACE-2007-1) is gratefully acknowledged.

\section{REFERENCES}

Aschwanden, M. J. 2001, ApJ, 560, 1035

D’Angelo, N. 1965, Phys. Fluids, 8, 1748

De Pontieu, B., McIntosh, S. W., Carlsson, M., et al. 2007, Science, 318, 1574

Halberstadt, G., \& Goedbloed, J. P. 1995, A\&A, 301, 559

Hansteen, V. N., Leer, E., \& Holtzer, T. E. 1997, ApJ, 482, 498

Hara, H., Tsuneta, S., Lemen, J. R., Acton, L. W., \& McTiernan, J. M. 1992, PASJ, 44, L135

Hollweg, J. V., \& Sterling, A. C. 1994, ApJ, 282, L31

Ionson, J. A. 1983, ApJ, 271, 778

Kadomtsev, B. B., \& Timofeev, A. V. 1963, Sov. Phys. Dokl., 7, 826

Klimtchuk, J. A. 2006, Sol. Phys., 234, 41

Litwin, C., \& Rosner, R. 1993, ApJ, 412, 375

Mandrini, C. H., Demoulin, P., \& Klimtchuk, J. A. 2000, ApJ, 530, 999

Mok, Y. 1987, A\&A, 172, 327

Moses, D., Clette, F., Delaboudinière, J.-P., et al. 1997, Sol. Phys., 175, 571

Narain, U., \& Ulmschneider, P. 1990, Space Sci. Rev., 54, 377

Ofman, L., Davila, J. M., \& Steinolfson, R. S. 1995, ApJ, 444, 471

Poedts, S., Belien, A. J. C., \& Goedbloed, J. P. 1994, Sol. Phys., 151, 271

Poedts, S., \& Goedbloed, J. P. 1997, A\&A, 321, 935

Poedts, S., Goossens, M., \& Kerner, W. 1989, Sol. Phys., 123, 83

Poedts, S., Goossens, M., \& Kerner, W. 1990, ApJ, 360, 279

Priest, E. R. 1982, Solar Magnetohydrodynamics (Dordrecht: Reidel)

Ruderman, M. S., Berghmans, D., Goossens, M., \& Poedts, S. 1997, A\&A, 320, 305

Saleem, H., Vranjes, J., \& Poedts, S. 2007, A\&A, 471, 289

Schrijver, C. J., Title, A. M., Berger, T. E., et al. 1999, Sol. Phys., 187, 261

Steinolfson, R. S., \& Davila, J. 1993, ApJ, 415, 354

Vranjes, J., \& Poedts, S. 2009a, Europhys. Lett., 86, 39001

Vranjes, J., \& Poedts, S. 2009b, MNRAS, 398, 918

Vranjes, J., \& Poedts, S. 2009c, MNRAS, 400, 2147

Weiland, J. 2000, Collective Modes in Inhomogeneous Plasma (Bristol: IOP Publishing) 